\title{
Estimation of Time-Dependent Reproduction Number for Global COVID-19 Outbreak
}

\author{
Tatiana Petrova ${ }^{1 *}$, Dmitry Soshnikov ${ }^{2,3}$ and Andrey Grunin ${ }^{1}$ \\ 1 Lomonosov Moscow State University, Faculty of Physics, Moscow, Russia \\ 2 Microsoft, Developer Relations, Moscow, Russia \\ 3 Higher School of Economics, Faculty of Computer Science, Moscow, Russia \\ *Correspondence: Tatiana Petrova, Faculty of Physics, Lomonosov Moscow State University, \\ 119992, Leninskie gory 1bld2, Moscow, Russia, Tel: +7 495939 12 50, e-mail: tapetrova@physics.msu.ru
}

\begin{abstract}
Real-time estimation of the parameters characterising infectious disease transmission is important for optimization quarantine interventions during outbreaks. One of the most significant parameters is the effective reproduction number - number of secondary cases produced by a single infection. The current study presents an approach for estimating the effective reproduction number and its application to COVID-19 outbreak. The method is based on fitting SIR epidemic model to observation data in a sliding time window and allows to show real-time dynamics of reproduction number at any phase of epidemic for countries globally. Online data on COVID-19 daily cases of infections, recoveries, deaths are used. Finally, time-dependent reproduction number is explored in connection with dynamics of peoples mobility. The method allows to assess the disease transmission potential and understand the effect of interventions on epidemics spread. It also can be easily adapted to future outbreaks of different pathogens. The tool is available online as Python code from the Github repository.
\end{abstract}

Keywords: reproduction number; infectious disease epidemiology; COVID-19; epidemic modelling; mobility index

\section{Introduction}

Coronavirus disease (COVID-19) was first identified in late December 2019 in Wuhan (China) and during the first half of 2020 spread all over the word. On March 11th the coronavirus outbreak was labelled a pandemic by the World Health Organization [1]. One of the key points during an epidemic is to design appropriate mathematical models and tools for effective decision-making [2]. For optimizing the quarantine steps it is important to estimate parameters characterizing infectious disease transmission using real-time data and track the temporal changes in those values [3].

The time-dependent (or effective) reproduction number $\left(R_{t}\right)$ is one of the key parameters characterizing evolution of an epidemic. The value of $R_{t}$ is a function of time, and it represents the expected number of secondary cases arising from a primary case infected at time $\mathrm{t}$ [4]. $R_{t}$ is affected by many factors, including social distancing and other quarantine steps, and changes during an outbreak. If $R_{t}<1$ the disease will decline and eventually die out. If $R_{t}>1$ the disease will be transmitted between people, and an outbreak is likely to occur. Reducing the reproduction number below one is one of the main goals of implementing quarantine steps [5]. In the report [6] a consistent correlation between reductions in mobility and reductions in transmission intensity of COVID-19 was found.

Different methods are available to define the initial reproduction number $R_{0}$ and time-dependent reproduction number $R_{t}$. The numerical estimations of reproduction number using different methods vary depending on implementation [4,7-12]. Some of the standard methods were applied to assume 
basic and time-dependent reproduction number for COVID-19 outbreak for different countries using different implementations [6, 13 -16].

In review [17] the differences for estimations of COVID-19 reproduction number for China are shown and mentioned that the different forecasts can be explained by insufficient data and different estimation techniques.

The useful open-source tool for estimating reproduction number in real-time, requiring only data that are commonly recorded during an outbreak (infections, recoveries, deaths, infection duration), with clear science based methodology and without limitations on epidemic phase will allow to forecast the progression of disease outbreaks and understand the effect of interventions on epidemics spread.

\section{Material and Methods}

To estimate the time-dependent reproduction number, the following procedure based on classical SIR model is used. The starting point at which the number of infected people in the country gets above 1000 is determined. We then move a sliding window with the width of 7 days over the whole period, and for each step we estimate the time-dependent reproduction number by minimizing square difference between real and predicted number of new infected cases. Finally, time-dependent reproduction number is explored in connection with dynamics of peoples mobility.

\subsection{Data Sources}

Real-time data on COVID-19 daily cases of infections, recoveries, deaths, as well as each country population are retrieved from the COVID-19 Data Repository by the Center for Systems Science and Engineering (CSSE) at Johns Hopkins University [18]. The data are reported by each country's official surveillance system from the January 22nd, 2020.

Data reflecting people mobility (mobility data) are sourced from Apple [19]. It is generated by counting the number of requests made to Apple Maps for directions. The data sets are compared to reflect a change in volume of people driving, walking or taking public transit around the world. Data availability in a particular city, country, or region is subject to a number of factors, including minimum thresholds for direction requests made per day [20]. The mobility data for China are temporarily not available. The data are available from the January 13th (except May, 11th-12th) and updated every day.

\subsection{Processing data}

\subsubsection{COVID-19 data}

The online data of numbers of infected, recovered, deceased people and each country population [18] are joined in one table and the dates are transposed, which gives us country dataframe. Then the numbers are smoothed out by computing 3-days rolling average on all the columns. The "Removed" column that contains the number of people removed from the susceptible population (i.e. recovered or deceased) is also computed. The analysis is based on countries which have at least 1000 infections reported in total.

\subsubsection{Apple mobility index data}

All available apple mobility data is collected and mean values of three data streams ('driving', 'walking' and 'transit') for each country are obtained. 7-days running average is applied to reduce weekly fluctuations.

\subsection{SIR modelling}

SIR modelling is a classical approach to epidemic simulation presented by Kermack-McKendrick [21] for the number of people infected with a contagious illness in a closed population over time. The assumptions of model are: constant (closed) population size $(N)$; constant rates (e.g., transmission, removal rates - the probability of disease transmission in a single contact multiplied by the average number of contacts per person); no demography (i.e., births and deaths); well-mixed population (where 
any infected individual has a probability of contacting any susceptible individual that is reasonably well approximated by the average).

The SIR model describes the connection between $S$ (susceptible - number of people who have the potential to be infected), I (infected - number of infected people), and $R$ (removed - number of people who are non susceptible to infection, this includes the number of deceased people as well) with the following system of ordinary differential equations (ODE):

$$
\begin{aligned}
& \frac{d S}{d t}=-\frac{\beta S I}{N}, \\
& \frac{d I}{d t}=\frac{\beta S I}{N}-\gamma I, \\
& \frac{d R}{d t}=\gamma I .
\end{aligned}
$$

$\beta$ is known as the effective contact rate. It is assumed that in a unit time each infected individual will come into contact with $\beta N$ people. From those people, the proportion of susceptible people is $S / N$, thus the speed at which new infections occur is considered to be $-\beta S I / N$.

$\gamma$ is the removal rate, and the number $1 / \gamma$ defines the number of days during which a person stays infected. Thus the term $\gamma I$ defines the speed at which infected individuals are moved from being infected to recovered (or deceased).

The rates are supposed to be constant. In the assumption that the population is completely susceptible $(S=N)$, the basic reproductive number is $R_{0}=\beta / \gamma$ [22].

\subsection{Determining parameters of the model}

Model predictions based on system (1) depend on the parameters $(\beta, \gamma)$. Optimization of the model with respect to parameters $\beta$ and $\gamma$ and fitting it to the real data allows to find the parameters that correspond to the actual outbreak. It is more consistent to optimize for $\beta$, and set the $\gamma$ parameter according to medical studies. According to WHO [23], the median time from onset to clinical recovery for mild COVID-19 cases is approximately 2 weeks, and it is 3-6 weeks for patients with severe or critical disease. Among patients who have died, the time from symptom onset to outcome ranges from 2 to 8 weeks. Since the time period is wide enough, we considered the mean recovery time of 30 days, and fixed $\gamma$ as $1 / 30$, respectively.

To smooth inaccuracies in the statistics at beginning of the outbreak the calculations start from day $t_{0}$, where $t_{0}$ is the first day when the number of infected people is above 1000:

$$
t_{0}=\min \{t \mid I(t)>1000\} .
$$

Considering one country at a time, the following denotations for the data are used: $V(t)$ - the number of total (accumulated) infected cases at day $t, t>t_{0}, E(t)$ - number of recovered cases at day $t$, $D(t)$ - number of fatal cases at day $t$.

The values corresponding to each $\beta$ are denoted as $S_{\beta}(t), I_{\beta}(t)$ and $R_{\beta}(t)$, respectively. $S_{\beta}(t), I_{\beta}(t)$ and $R_{\beta}(t)$ are the solutions of system (1) with initial values of:

$$
\begin{aligned}
& S\left(t_{0}\right)=N-V\left(t_{0}\right)-D\left(t_{0}\right)-E\left(t_{0}\right), \\
& I\left(t_{0}\right)=V\left(t_{0}\right) \\
& R\left(t_{0}\right)=D\left(t_{0}\right)+E\left(t_{0}\right) .
\end{aligned}
$$

Here $N$ is the population of a country being considered.

The accumulated number of total infected people computed by the model is given by:

$$
I_{\beta}^{+}(t)=I_{\beta}(t)+R_{\beta}(t),
$$

and new total infected cases each day is equal to

$$
I_{\beta}^{\prime}(t)=I_{\beta}^{+}(t+1)-I_{\beta}^{+}(t) \text {. }
$$

The actual number of daily new infected cases

$$
V^{\prime}(t)=V(t+1)-V(t) \text {. }
$$

The value $\beta^{*}$ that corresponds to the minimal discrepancy between $V^{\prime}(t)$ and $I_{\beta}{ }^{\prime}(t)$ in the 7-day period is defined below (we will denote the number of days in consideration by $n=7$ ): 


$$
\beta^{*}=\operatorname{argmin}_{\beta} \sum_{t=t_{0}}^{t_{0}+n}\left(V^{\prime}(\mathrm{t})-I_{\beta}^{\prime}(\mathrm{t})\right)^{2} .
$$

The process of finding argmin (the minimum value along a given axis) is a complex optimization process, and at each step the numerical solution of ODE (1) is needed.

Powell's method (Powell's conjugate direction method) is used for optimization because this algorithm does not rely on gradients and is fast enough [24].

Having obtained $\beta^{*}$, we calculate $R_{0}$ as follows:

$$
R_{0}=\beta^{*} / \gamma .
$$

\subsection{Application of SIR modelling for time-dependent reproduction number estimation}

Similar to the basic reproductive number $R_{0}$ that is a characteristic of a disease, the time-dependent reproductive number $R_{t}$ is considered. $R_{t}$ dynamics during the pandemic takes into account isolation measures and the proportion of nonsusceptible population, and can be used to estimate the effectiveness of quarantine measures.

The same approach is followed for estimating $R_{\mathrm{t}}$ in real time:

Start from the point at which there are 1000 infected people.

Move a sliding window of width $\mathrm{n}(n=7$ is used) over the whole period till present day.

At each point, $n$ consecutive days are used to estimate $\beta$ (and, thus, $R_{t}$ ) by minimizing the square difference between the real and predicted number of new infected people.

\section{Results}

The approach described above allows to find real-time dynamics of reproduction number and easily analyze it in connection with other factors directly affecting the epidemic spread (number of daily infected cases, people's mobility level, rate of changes in people's mobility level). The results are presented in Figs.1-4 for 12 countries and can be extended to any country in the world reported in [18] and [19].

\subsection{Comparison of reproduction number dynamics for different countries}

General trends in the COVID-19 pandemic spread connected with $R_{t}$ dynamics in different countries are described in Fig. 1. The following main trends may be identified:

- The $R_{t}$ values decreased significantly compared to the initial value for all considered countries.

- For most of considered countries $R_{t}$ has already dropped below 1, which is consistent with public statements [25-27].

- Countries are characterized by different $R_{t}$ shapes - the $R_{t}$ curves have different initial values and different decrease rates.

- Decrease of $R_{t}$ in time is not always monotonous. Based on the graphs, artifacts (spikes) associated with delayed statistics (France, China) are observed, as well as those one caused by quarantine events (Russia, India, Brazil). 

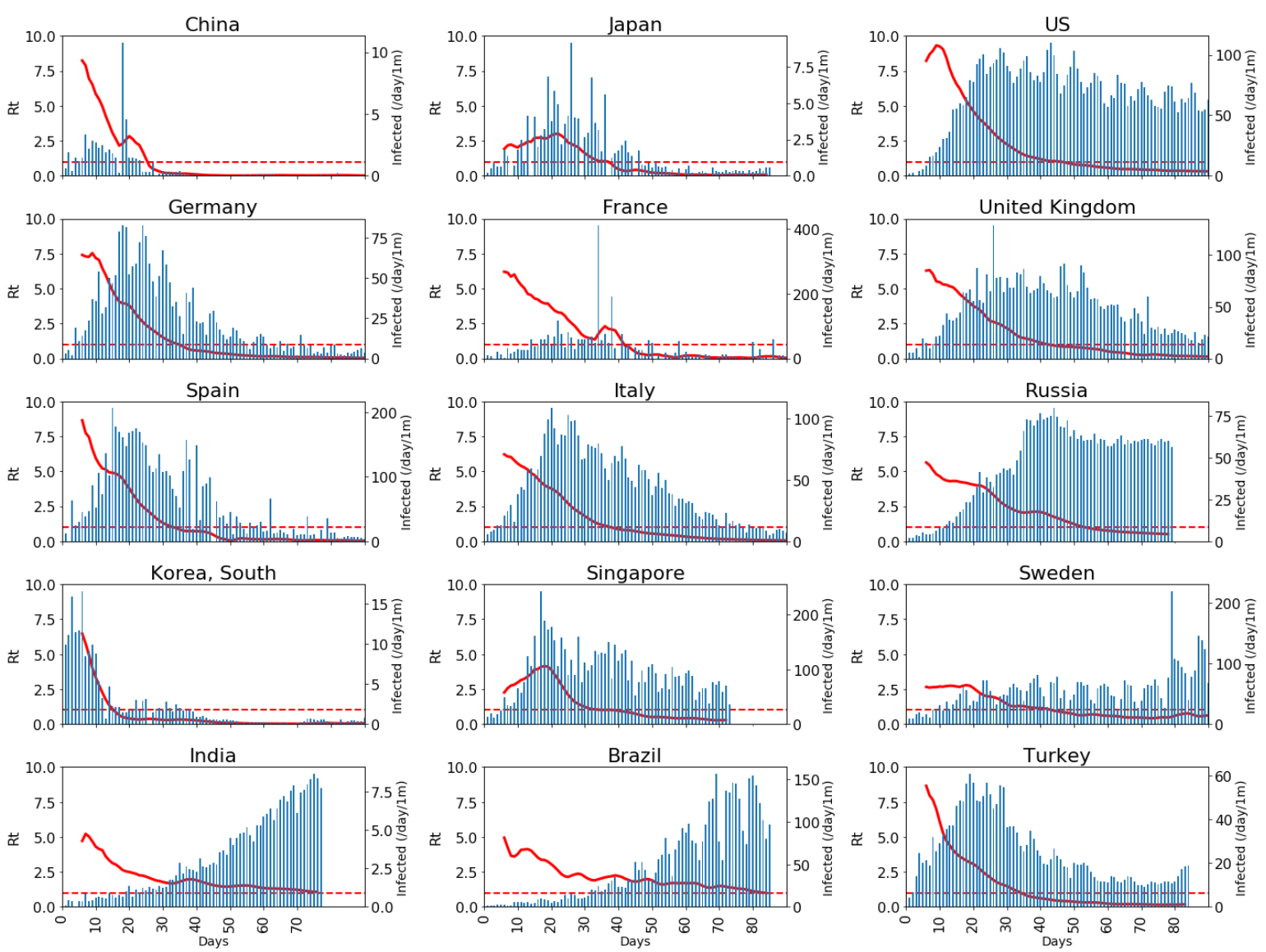

Figure 1. Time-dependent reproductive number $R_{t}$ (red line, left y-axis) and daily number of infected normalized by country population for each country (blue histogram, right $y$-axis). On the x-axis the number of days since the first 1000 infected cases is shown, which makes it possible to compare the speed of the epidemic spread regardless of the actual timeframe. The number of new cases is measured in cases per million of people. Normalized number of new cases is shown on different scales for each graph on purpose, to make the shape of the graph more visible. On all graphs the period from 0 to 90 days is shown, therefore for some of the countries the graph ends earlier.

Fig. 2 illustrates the points above in a more visible way.

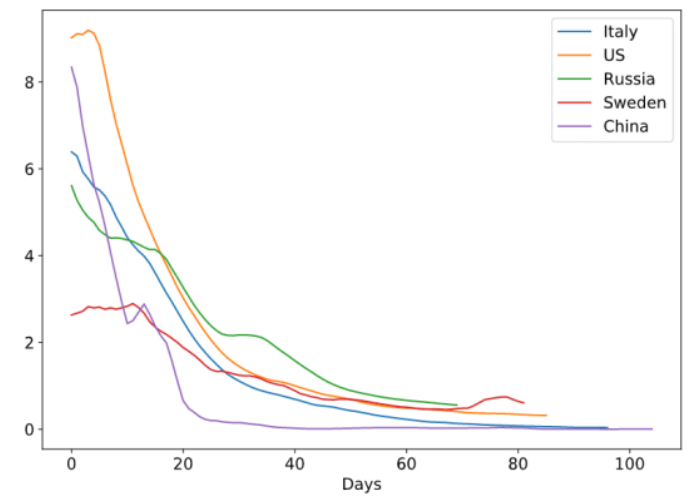

(a)

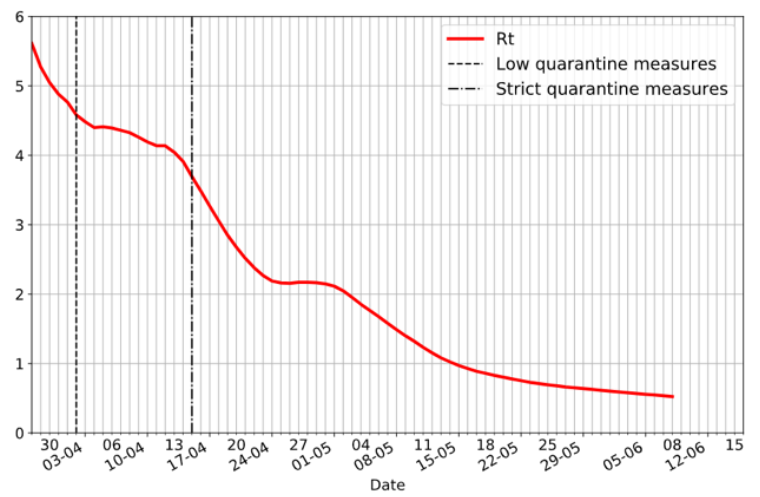

(b)

Figure 2. Time dynamics of time-dependent reproductive number $R_{t}$. (a) Comparison of $R_{t}$ shapes for different countries, on the x-axis the number of days since the first 1000 infected cases is shown. (b) Comparison of $R_{t}$ shape for Russia with key quarantine measures marked. 
The differences of $R_{t}$ curve shapes for Italy, US, Russia, Sweden and China are shown. In the beginning of the outbreak the US and China had the highest values of reproductive number and Sweden - the lowest one. But the rate of $R_{t}$ decrease was the fastest for China, and the slowest for Sweden and Russia.

The shape of $R_{t}$ for Russia is presented in more detail on Fig.2 (b). Fig. 2 (b) indicates two dates when isolation measures were put in place in Russia:

- On the April, 2nd government announced the second wave of quarantine measures (more strict than just recommended self-isolation, but still low enough);

- On April, 15th mandatory the quarantine measures became strict - permits for living houses were introduced in Moscow and a few other large cities.

In order to get the relationship of people's mobility with reproduction number, and automatically track this connection daily for different countries, the approach developed in this article was supplemented by visualizing Apple Mobility Index.

\subsection{Relation between Apple mobility index and reproduction number dynamics}

Relationships between $R_{t}$ and the mobility index in different countries are presented in Fig. 3 . It is supposed that this Mobility Index reflects the level of lockdown.
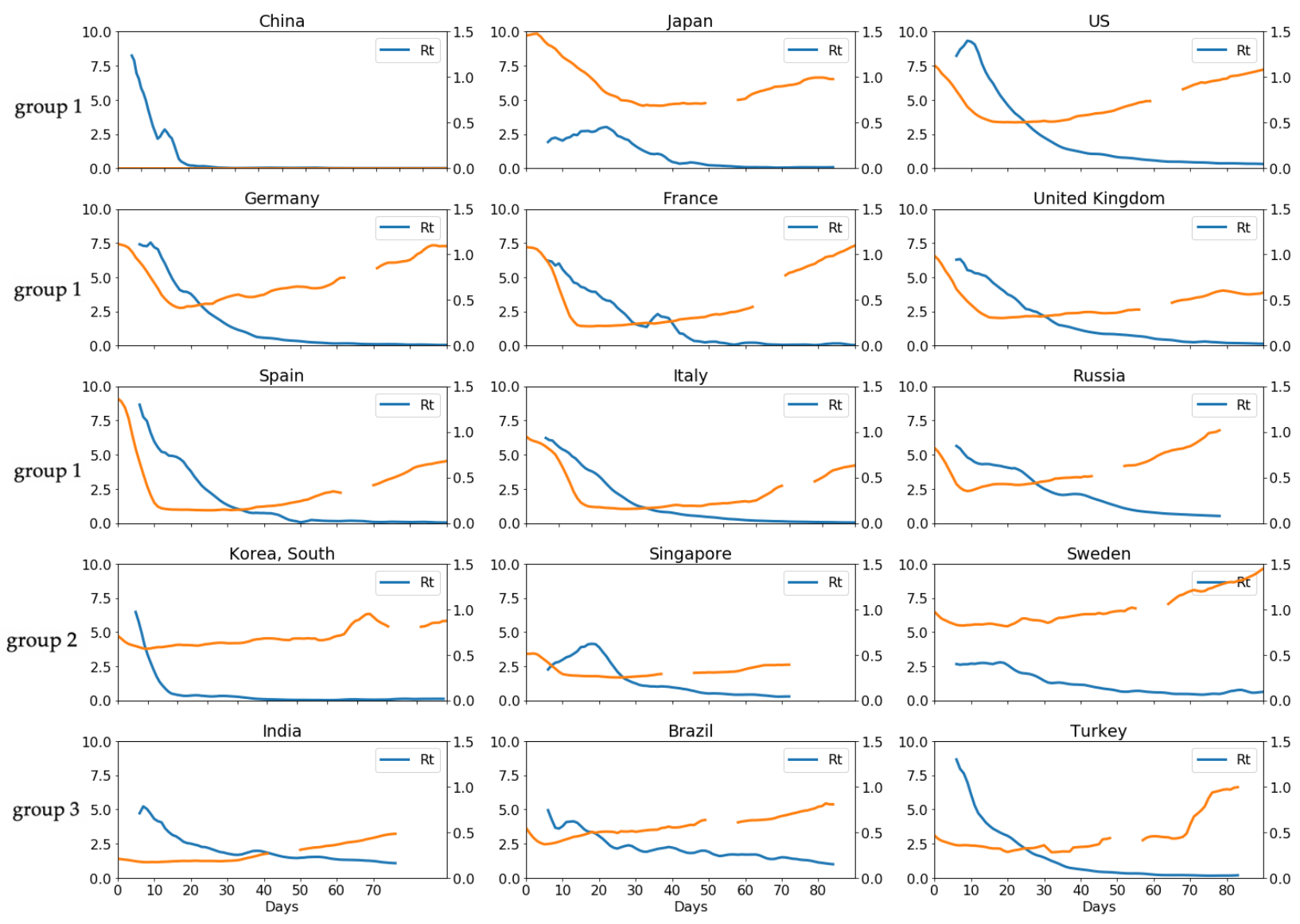

Figure 3. Relationship between Apple mobility index (orange line, right y-axis) and time-dependent reproductive number $R_{t}$ (blue line, left y-axis). Time-dependent reproductive number $R_{t}$ and Apple mobility index (aggregated as mean of three data streams: 'driving', 'walking' and 'transit' mobility) smoothed by 7-days sliding window are shown. On the x-axis the number of days since the first 1000 infected cases; $x$-axis and $y$-axis are shown in the same scale for all countries. The mobility data for China is temporarily not available.

There is a consistent correlation between reduction in mobility level and reduction in reproduction number values. The correlation is in good agreement with the results of [6]. Fig.3 also separates countries by different types of quarantine measures. 
The first group contains countries that applied lockdown to reduce the epidemic spread. Japan, United States, Germany, France, United Kingdom, Spain, Italy and Russia demonstrate rapid reduction in the mobility index by implementation of strict quarantine measures. The mobility data for China temporarily not available, but lockdown in China was called by WHO as "unprecedented in public health history" and allowed to stop the spread of coronavirus in China.

The second group are countries without lockdown. South Korea and Singapore introduced one of the largest and best-organised epidemic control programs in the world. Different measures have been taken to screen the mass population for the virus, and isolate any infected people as well as trace and quarantine those who contacted them, without further lockdown [28]. Sweden has not imposed a lockdown too, for citizens it was recommended to follow a series of recommendations from the government agency, but without tracing contacts of those infected [29]. It is seen that Swedish model is much less effective than South Korea's one, though in the beginning of the outbreak, the reproductive number for Sweden was only 2.6 because of high social distancing specific for Scandinavian countries.

For the third group of countries (India, Brazil and Turkey) the mobility index was relatively low initially, and quarantine measures did not have any significant impact on its value. So it is difficult to evaluate measures and their impact on epidemic spread.

\subsection{Relation between derivative of reproduction number and Apple mobility index}

To assess the relation between the dynamics of $R_{t}$ and the level of mobility, the derivative of $R_{t}$ in relation to the Apple mobility index was considered (Fig. 4). It can be seen that the highest rate of decrease in $R_{t}$ correlates with the minimum of mobility index.

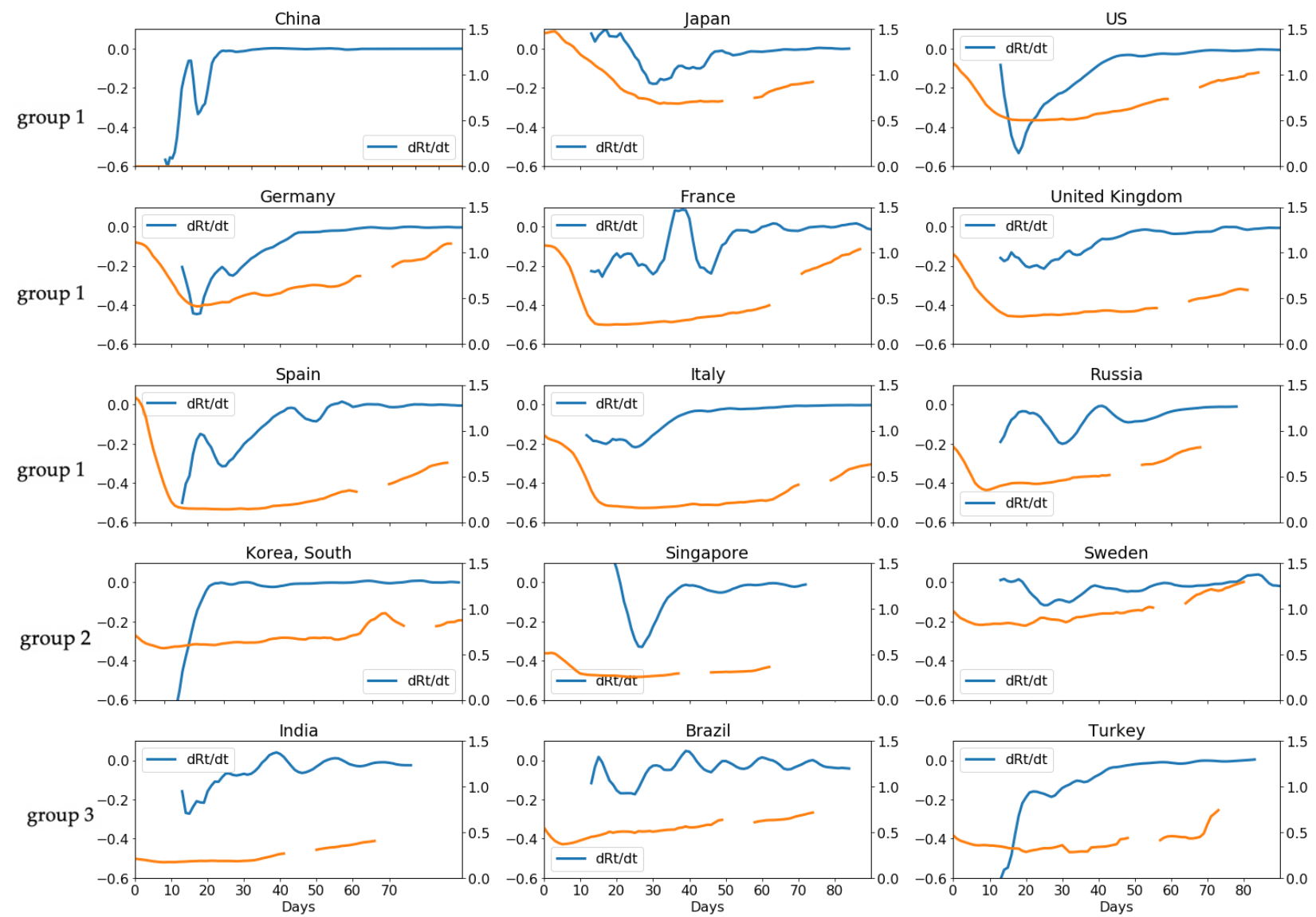

Figure 4. Relationship between Apple mobility index (orange line, right y-axis) and time derivative of time-dependent reproductive number $R_{t}$ (blue line, left y-axis). Derivative of time-dependent reproductive number $R_{t}$ and smoothed Apple mobility index (aggregated as mean of three data streams: 'driving', 'walking' and 'transit' mobility) are shown. On the $x$-axis the number of days since the first 1000 infected cases; $x$-axis and $y$-axis are shown in the same scale for all countries. The mobility data for China is temporarily not available. 
The non-monotonous curve of $R_{t}$ derivatives with monotonous mobility curve suggests that the reproduction number is influenced by mobility and non-mobility events (for example, massive violations of social distancing). Local extrema of the $R_{t}$ derivative graph allow us to suggest the dates of such events. For all groups of countries, the mobility index trend matches the $R_{t}$ derivative trend in general. It clearly shows that after removal of quarantine restrictions, the rate of $R_{t}$ growth is increasing.

\section{Discussion}

There are some restrictions regarding the results of calculations.

First of all, we do not separate internal and external cases, assuming implicitly that all incident cases after the first time-point arise from local transmission, i.e. it does not account for the possibility that cases (other than those appearing at the first timestep) are imported from other locations or derived from alternative host species. This assumption is used, for example, by [30]. In our case, it is applicable since we try to calculate the potential velocity of epidemic spreading and the way of infection appearance is not a case of the study.

Based on previous studies, we also suspect that recovered person can not become susceptible again, after having already recovered from the infection [31].

We do not formally distinguish the non-diagnosed individuals, who spread the infection more because they are not in isolation, and diagnosed individuals, who transmit the disease much less thanks to proper isolation and complying with strict rules, either in hospital or at home. To make predictions more accurate, it makes sense to use more accurate model that accounts for incubation period, such as SEIR model [32]. However, using more accurate models is likely not to have a huge impact on the resulting numbers, by may complicate the optimization process with limited number and accuracy of data points.

\section{Conclusions}

The model for calculating the time-dependent reproduction number $\left(R_{t}\right)$ in real-time, based on classical SIR approach, without limitations on epidemic phase and implemented to countries globally based on publicly available data was provided.

The useful open-source tool for estimating $R_{t}$ in real-time, based on this model, requiring only data that are commonly recorded during an outbreak (infections, recoveries, deaths, infection duration), with clear scientific methodology and without limitations on epidemic phase allows to forecast the progression of disease outbreaks and understand the effect of interventions on epidemics spread. This tool also allows to assess the disease transmission potential, understand the effect of interventions on epidemics spread and can be easily adapted to future outbreaks of different pathogens.

For COVID-19 spread it is shown that decrease in $R_{t}$ value correlates with level of quarantine measures for group of countries with lockdown and the highest rate of decrease in $R_{t}$ correlates with the minimum of mobility index.

The tool is available online as Python code from the Github repository.

\section{Supplementary data}

A tool for estimating the time-dependent reproduction number during SARS-COVID-19 pandemic for countries worldwide is available online as Python code from the following Github repository: https://github.com/shwars/SlidingSir

\section{Funding}

This work was supported as part of the research program of MSU Center for Storage and Analysis of Big Data.

\section{Acknowledgments}

The authors would like to thank the Center for Systems Science and Engineering (CSSE) at Johns Hopkins University and Apple Inc. for useful data collection services. 


\section{References}

1. WHO Director-General's opening remarks at the media briefing on COVID-19 - 11, 11 March 2020. Available from: https://www.who.int/dg/speeches/detail/who-director-general-s-opening-remarks-at-the-mediabriefing-on-covid-19---11-march-2020 (accessed on 17 of June 2020).

2. J.A. Polonsky, A. Baidjoe, Z.N. Kamvar, A. Cori, K. Durski, W.J. Edmunds, R.M. Eggo, S. Funk, L. Kaiser, P. Keating, et al. Outbreak analytics: a developing data science for informing the response to emerging pathogens Philos. Trans. R. Soc. B, 374 (2019), Article 20180276. doi: 10.1098/rstb.2018.0276.

3. R.N. Thompson, C.A. Gilligan, N.J. Cunniffe Control fast or control smart? When should invading pathogens be controlled? PLoS Comp. Biol., 14 (2018), Article e1006014 https://doi.org/10.1371/journal.pcbi.1006014

4. R.N. Thompson, J.E. Stockwin, R.D. van Gaalen, J.A. Polonsky, Z.N. Kamvar, P.A. Demarsh, E. Dahlqwist, S. Li, E. Miguel, T. Jombart, J. Lessler, S. Cauchemez, A. Cori, Improved inference of time-varying reproduction numbers during infectious disease outbreaks, Epidemics, Volume 29, 2019, 100356, ISSN 1755-4365, https://doi.org/10.1016/j.epidem.2019.100356.

5. A. Kucharski, A. Camacho, Y. Aki-Sawyerr, M.A. White, S. Flasche, M. Baguelin, T. Pollington, J.R. Carney, R. Glover, E. Smout, A. Tiffany Temporal changes in Ebola Transmission in Sierra Leone and implications for control requirements: a real-time modelling study PLoS Curr., 7 (2015), pp. 1-19. doi: 10.1371/currents.outbreaks.406ae55e83ec0b5193e30856b9235ed2

6. Pierre Nouvellet, Sangeeta Bhatia, Anne Cori, et. al. Report 26 Reduction in mobility and COVID-19 transmission, 08 June 2020. Available from: https:/www.imperial.ac.uk/media/imperialcollege/medicine/mrc-gida/2020-06-08-COVID19-Re port-26.pdf (accessed on 17 of June 2020).

7. Wallinga J, Lipsitch M: How generation intervals shape the relationship between growth rates and reproductive numbers. Proceedings of the Royal Society B: Biological Sciences. 2007, 274: 599-10. doi: 1098/rspb.2006.3754.

8. Chowell G, Viboud C, Simonsen L, Miller MA, Acuna-Soto R, Díaz J, Martínez-Martín AF: The 1918-19 Influenza Pandemic in Boyacá. Colombia. Emerging infectious diseases. 2012, 18: 48-56. doi: 10.3201/eid1801.101969

9. Boëlle PY, Bernillon P, Desenclos JC: A preliminary estimation of the reproduction ratio for new influenza A(H1N1) from the outbreak in Mexico, March-April 2009. Euro Surveill. 2009, 14 (19): pii=19205. https://doi.org/10.2807/ese.14.19.19205-en

10. Nishiura H, Wilson N, Baker M: Estimating the reproduction number of the novel influenza A virus (H1N1) in a Southern Hemisphere setting: preliminary estimate in New Zealand. N. Z. Med. J. 2009, 122: 73-77.

11. Obadia, T., Haneef, R. \& Boëlle, P. The R0 package: a toolbox to estimate reproduction numbers for epidemic outbreaks. BMC Med Inform Decis Mak 12, 147 (2012). https://doi.org/10.1186/1472-6947-12-147

12. Anne Cori, Neil M. Ferguson, Christophe Fraser, Simon Cauchemez, A New Framework and Software to Estimate Time-Varying Reproduction Numbers During Epidemics, American Journal of Epidemiology, Volume 178, Issue 9, 1 November 2013, Pages 1505-1512, https://doi.org/10.1093/aje/kwt133

13. Niket Thakkar, Roy Burstein, Daniel Klein, and Mike Famulare. Sustained reductions in transmission have led to declining COVID19 prevalence in King County, WA. Results as of 10 a.m. on April 29, 2020. Available from:

https://covid.idmod.org/data/Sustained_reductions_in_transmission_have_led_to_declining_COVID_19_pre valence_in_King_County_WA.pdf (accessed on 17 of June 2020).

14. Giordano, G., Blanchini, F., Bruno, R. et al. Modelling the COVID-19 epidemic and implementation of population-wide interventions in Italy. Nat Med (2020). https://doi.org/10.1038/s41591-020-0883-7

15. Wangping Jia, Ke Han, Yang Song, Wenzhe Cao, Shengshu Wang, Shanshan Yang, Jianwei Wang, Fuyin Kou, Penggang Tai, Jing Li, Miao Liu, Yao He. Extended SIR Prediction of the Epidemics Trend of COVID-19 in Italy and Compared With Hunan, China. Frontiers in Medicine. 7. 2020.169 doi: 10.3389/fmed.2020.00169

16. Yaset Caicedo-Ochoa, David E. Rebellón-Sánchez, Marcela Peñaloza-Rallón, Hector F. Cortés-Motta, Yardany R. Méndez-Fandiño, Effective Reproductive Number estimation for initial stage of COVID-19 pandemic in Latin American Countries, International Journal of Infectious Diseases, Volume 95, 2020, Pages 316-318, ISSN 1201-9712 https://doi.org/10.1016/j.ijid.2020.04.069.

17. Rafiq, D, Batool, A, Bazaz, MA. Three months of COVID-19: A systematic review and meta-analysis. Rev Med Virol. 2020; e2113. https://doi.org/10.1002/rmv.2113

18. COVID-19 Data Repository by the Center for Systems Science and Engineering (CSSE) at Johns Hopkins University. Available from: https://github.com/CSSEGISandData/COVID-19 (accessed on 17 of June 2020). 
19. Mobility Trends Reports. Available from: http://apple.com/covid19/mobility

20. Newsroom: Apple makes mobility data available to aid COVID-19 efforts. April 14, 2020. Available from: https://www.apple.com/newsroom/2020/04/apple-makes-mobility-data-available-to-aid-covid-19-efforts/ (accessed on 17 of June 2020).

21. Kermack, W. O. and McKendrick, A. G. (1933). Contributions to the mathematical theory of epidemics, iii further studies of the problem of endemicity. Proceedings of the Royal Society of Edinburgh. Section A. Mathematics. 141 94-122.

22. James Holland Jones. Notes On R0. May 1, 2007. https://web.stanford.edu/ jhj1/teachingdocs/Jones-on-R0.pdf (accessed on 17 of June 2020).

23. Report of the WHO-China Joint Mission on Coronavirus Disease 2019 (COVID-19),16-24 February 2020. Available from: https://www.who.int/docs/default-source/coronaviruse/who-china-joint-mission-on-covid19-final-report.pdf (accessed on 17 of June 2020).

24. Powell, M.. (2007). A View of Algorithms for Optimization Without Derivatives. Mathematics TODAY. 43. http://www.damtp.cam.ac.uk/user/na/NA_papers/NA2007_03.pdf (accessed on 17 of June 2020).

25. Germany's 'R-Naught': Are Three Days Over 1.0 Cause for Worry? Available from: https://www.nytimes.com/2020/05/12/world/europe/germany-coronavirus-r-number.html (accessed on 17 of June 2020).

26. Most of Spain has virus contagion ' $R$ ' number below one: health official. Available from: https://www.reuters.com/article/us-health-coronavirus-spain-contagion/most-of-spain-has-virus-contagionr-number-below-one-health-official-idUSKBN22B1CN (accessed on 17 of June 2020).

27. Russia's coronavirus reproduction rate falls to 0.95 . Available from: https://tass.com/society/1161729

28. What We Can Learn From Singapore, Taiwan and Hong Kong About Handling. Coronavirus Available from: https://time.com/5802293/coronavirus-covid19-singapore-hong-kong-taiwan/ (accessed on 17 of June 2020).

29. In the Coronavirus Fight in Scandinavia, Sweden Stands Apart. Available from: https://www.nytimes.com/2020/03/28/world/europe/sweden-coronavirus.html

30. Anne Cori, Neil M. Ferguson, Christophe Fraser, Simon Cauchemez, A New Framework and Software to Estimate Time-Varying Reproduction Numbers During Epidemics, American Journal of Epidemiology, Volume 178, Issue 9, 1 November 2013, Pages 1505-1512. https://doi.org/10.1093/aje/kwt133

31. Lan, L. et al. Positive RT-PCR test results in patients recovered from COVID-19. JAMA (2020). https://doi.org/10.1001/jama.2020.2783

32. Prem, Kiesha \& Liu, Yang \& Russell, Timothy \& Kucharski, Adam \& Eggo et.al. (2020). The effect of control strategies to reduce social mixing on outcomes of the COVID-19 epidemic in Wuhan, China: a modelling study. The Lancet Public Health. 5. 10.1016/S2468-2667(20)30073-6. doi: https://doi.org/10.1016/S24682667(20)30073-6 